\title{
Indirect immunofluorescence (IIF) of normal washed peripheral blood cells to demonstrate antineutrophil cytoplasmic antibodies (ANCA)
}

\author{
B Paspaliaris, M Pamio, J Savige
}

\begin{abstract}
Background-The "International consensus document on testing and reporting of antineutrophil cytoplasmic antibodies (ANCA)" requires all sera to be examined by indirect immunofluorescence (IIF). However, commercial neutrophil slides are expensive, fluorescence patterns can be difficult to interpret, and coincidental antinuclear antibodies (ANA) cannot be demonstrated; in addition, in house cytospin neutrophil preparations are time consuming to prepare and deteriorate with time.

Aims-To compare the IIF demonstration of ANCA, using washed peripheral blood cell smears, with commercial neutrophil preparations and with ANCA positivity as demonstrated by enzyme linked immunosorbent assay (ELISA).

Methods-Serum fluorescence positivity, pattern, and intensity using washed peripheral blood cell smears were compared with the results obtained using commercial neutrophil slides (INOVA). Fluorescence positivity, pattern, and intensity of 500 sera from consecutive patients with suspected vasculitis tested with washed peripheral blood cells were compared with binding in ELISAs for proteinase 3 (PR3) and myeloperoxidase (MPO).
\end{abstract}

Results-IIF of washed peripheral blood cell smears detected seven of eight sera with cytoplasmic fluorescence (C-ANCA), and 11 of 12 sera with perinuclear fluorescence (P-ANCA) demonstrated using commercial slides. The two sera that were negative by IIF were also negative in the ELISAs for both PR3-ANCA and MPOANCA. Of the 500 sera examined, there were 35 (7\%) with C-ANCA, 65 (13\%) with P-ANCA, and eight (2\%) IIF negative sera that were positive by either ELISA. There was a strong correlation between C-ANCA fluorescence and PR3-ANCA values $(\mathrm{p}<0.0001)$, and a moderate to strong correlation between P-ANCA fluorescence and MPO-ANCA values $(p<0.001)$ when ANCA fluorescence was demonstrated with washed peripheral blood cell smears.

Conclusions-Washed peripheral blood cells are a convenient and useful low cost alternative to commercial or cytospin neutrophil preparations for the IIF demonstration of ANCA.

(f Clin Pathol 2000;53:774-777)
Keywords: antineutrophil cytoplasmic antibodies; autoantibodies; indirect immunofluorescence

The most common reasons to request a test for antineutrophil cytoplasmic antibodies (ANCA) are to diagnose and to monitor inflammatory activity in the primary systemic small vessel vasculitides, Wegener's granulomatosis, microscopic polyangiitis, and ChurgStrauss syndrome. ${ }^{1-3}$ In these diseases, ANCA produce cytoplasmic (C-ANCA) or perinuclear (P-ANCA) neutrophil staining on indirect immunofluorescence (IIF) examination, and bind in enzyme linked immunoassays (ELISAs) for proteinase 3 (PR3) or myeloperoxidase (MPO).

The "International consensus statement on testing and reporting of antineutrophil cytoplasmic antibodies (ANCA)" requires all sera from patients suspected of having a small vessel vasculitis to be tested by a standardised IIF technique. ${ }^{5}$ Any sera with neutrophil cytoplasmic fluorescence or an antinuclear antibody (ANA) that could mask an ANCA should be tested in ELISAs for both PR3-ANCA and MPO-ANCA. ${ }^{4}$ (Optimally all sera should be tested by IIF and in both ELISAs.) Other recommendations include the titration of all sera positive only by IIF, or if any other cytoplasmic fluorescence or an ANA is present, and the inclusion of the most recent positive serum in the IIF (or ELISA) studies to demonstrate a change in antibody concentration.

Most laboratories in Australia currently use commercial neutrophil slides for IIF, and adopting the minimal requirements or the optimal recommendations of the consensus statement means that ANCA testing is expensive. We describe here a method using washed peripheral blood cell smears to demonstrate ANCA fluorescence, and we have compared results using these preparations with commercial neutrophil slides, and with ANCA positivity demonstrated by ELISA. The method has been modified from the technique formerly used in the detection of ANA, and in the first descriptions of ANCA.

\section{Methods}

IIF USING WASHED PERIPHERAL BLOOD CELLS Aliquots of $2 \mathrm{ml}$ of venous blood (of any $\mathrm{ABO}$ and $\mathrm{Rh}$ type) were collected into EDTA, and centrifuged at $1500 \times g$ at room temperature for six minutes. A washing step where $90 \%$ of the plasma was removed and replaced with phosphate buffered saline (PBS) was performed to reduce background fluorescence. The washed 
cells were then resuspended and smears made; these were air dried for five minutes, fixed with $100 \%$ ethanol at $-20^{\circ} \mathrm{C}$ for five minutes, and fan dried for 10 minutes. A circle was marked on the thin end of the smear.

Test sera were diluted 1/10 in PBS and incubated within the circle for 15 minutes at room temperature. Slides were then washed five times in PBS, incubated for a further 15 minutes with $1 / 500$ fluorescein conjugated $\mathrm{F}\left(\mathrm{ab}^{\prime}\right)_{2}$ fragment of polyclonal antiglobulin (AMRAD, Melbourne, Australia) in PBS, washed, and examined by epi-illumination in a fluorescent microscope. The fluorescence was described as C-ANCA, C-ANCA (atypical), $\mathrm{P}$-ANCA, and atypical, as described in the International consensus statement. ANA were noted. These smears could be stored at $-20^{\circ} \mathrm{C}$ for one to two weeks, and two sera could be tested separately on a single smear.

COMPARISON OF IIF POSITIVITY AND INTENSITY USING COMMERCIAL NEUTROPHIL PREPARATIONS AND WASHED PERIPHERAL BLOOD CELLS

Sera with C-ANCA or P-ANCA demonstrated on commercial (INOVA) neutrophil slides and with known PR3-ANCA and MPO-ANCA positivity were examined using washed peripheral blood cell smears.

COMPARISON OF ANCA POSITIVITY BY IIF OF PERIPHERAL BLOOD SMEARS AND IN ELISAS FOR PR3 AND MPO-ANCA

Five hundred sera from consecutive patients suspected of having a systemic vasculitis were tested by both IIF and in ELISAs for both PR3-ANCA and MPO-ANCA, according to the optimal recommendations of the International consensus statement. The assay for PR3-ANCA (Calbiochem, San Diego, California USA) was performed according to the manufacturer's instructions. The normal range was $<10 \mathrm{U}$. An in house assay for MPOANCA used MPO from Calbiochem, a 1/50 dilution of serum, and a 1/400 dilution of alkaline phosphatase linked anti-IgG (AMRAD); normal values were $<2 \mathrm{U}$.

Positive sera were diluted and the IIF titre on washed peripheral blood cells compared with the amount of binding in the PR3-ANCA and MPO-ANCA ELISAs.

\section{Results}

COMPARISON OF IIF POSITIVITY AND INTENSITY USING COMMERCIAL NEUTROPHIL PREPARATIONS AND WASHED PERIPHERAL BLOOD CELLS 1

Peripheral blood neutrophils were larger than the neutrophils in the commercial slides, and it was easier to distinguish between the different fluorescent patterns (fig 1).

Eight C-ANCA positive sera with intensity $1+$ to $3+$ were identified using commercial neutrophil slides, and three of these were PR3ANCA positive. Seven of these sera were also positive using washed peripheral blood cells. The serum that gave C-ANCA $1+$ to $2+$ results using commercial slides, but which was negative with peripheral blood smears, was from a patient with clinically inactive Wegener's granulomatosis, and was negative for both
PR3-ANCA and MPO-ANCA by ELISA. This serum was not tested for ANCA with other antigen specificities.

Twelve sera were P-ANCA positive using commercial neutrophil slides. Again, the fluorescence intensity varied from $1+$ to $3+$, and eight were MPO-ANCA positive. Eleven of these sera were also positive using washed peripheral cells. The serum that was P-ANCA $1+$ using the commercial slides, but negative with peripheral blood smears, was from a patient with a frusemide induced drug reaction, and was negative for both PR3-ANCA and MPO-ANCA by ELISA.

There was a good correlation between fluorescence intensity with the commercial slides and peripheral blood smears for both C-ANCA and P-ANCA: the weakest sera were weakest in both preparations, and the strongest were strongest in both.

Two of the sera containing P-ANCA had coincidental ANA on the peripheral blood neutrophil preparations with a screening dilution of $1 / 10$, but these ANA were not present when tested at a 1/40 dilution on HEp2 cells.

COMPARISON OF ANCA POSITIVITY BY IIF OF PERIPHERAL BLOOD SMEARS AND IN ELISAS FOR PR3 AND MPO-ANCA

In the 500 sera, there were $35 \mathrm{C}$-ANCA positive sera $(7 \%), 65$ P-ANCA positive sera $(13 \%)$, as well as eight IIF negative sera $(2 \%)$ that were positive by PR3-ANCA ELISA $(n=3)$ or MPO-ANCA ELISA $(n=5)$. No C-ANCA (atypical) or atypical IIF patterns were reported.

There was a strong correlation between the C-ANCA titre demonstrated by IIF of washed blood cells and PR3-ANCA values $(r=0.75$; $\mathrm{p}<0.0001$, by Spearman's rank correlation), and a moderate to strong correlation between P-ANCA titre and MPO-ANCA values $(r=0.55 ; \mathrm{p}<0.001)$ (fig 2$)$. Nine of the 35 C-ANCA positive sera were negative for PR3ANCA, and 35 of the 65 P-ANCA positive sera were negative for MPO-ANCA.

\section{Discussion}

The use of washed peripheral blood cell smears for IIF screening for ANCA is an effective and low cost alternative to the use of commercial neutrophil slides. The neutrophils are larger in these smears than in commercial preparations, making it easier to distinguish between cytoplasmic, perinuclear, and other fluorescence patterns. In addition, the smears contain lymphocytes and thus can demonstrate coincidental ANA when there is lymphocyte as well as neutrophil nuclear fluorescence. Washed peripheral cell smears are also convenient, because urgent single specimens can be tested without the other wells in a commercial multiwell slide being wasted. Finally, this method can be used in research involving animals where species specific blood smears will detect ANCA directed against antigenic epitopes that might differ between species. Although in house cytospin preparations of buffy coat ${ }^{6}$ or dextran sedimented neutrophils also result in larger cells than commercial preparations, and 

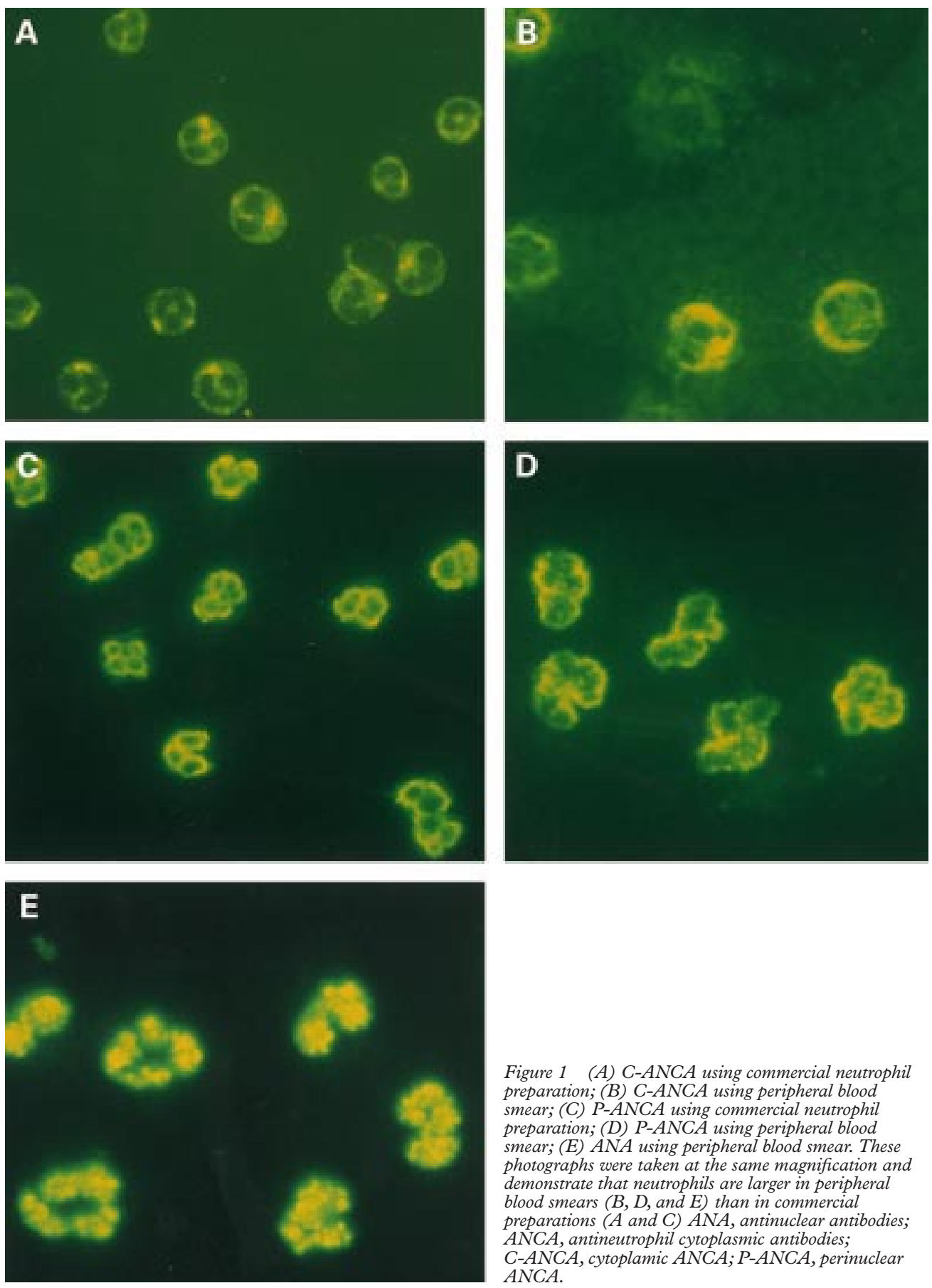

Figure 1 (A) C-ANCA using commercial neutrophil preparation; (B) C-ANCA using peripheral blood smear; (C) P-ANCA using commercial neutrophil preparation; (D) P-ANCA using peripheral blood smear; (E) ANA using peripheral blood smear. These photographs were taken at the same magnification and demonstrate that neutrophils are larger in peripheral blood smears $(B, D$, and $E)$ than in commercial preparations ( $A$ and $C$ ) $A N A$, antinuclear antibodies; ANCA, antineutrophil cytoplasmic antibodies; $C-A N C A$, cytoplamic ANCA; $P-A N C A$, perinuclear $A N C A$.

contain lymphocytes, these preparations deteriorate rapidly and unlike the peripheral blood smears are labourious to prepare.

Washed peripheral blood cell smears detected about $90 \%$ of the sera that were IIF positive with commercial neutrophil preparations. The sera that were negative by IIF on the washed smears were also negative in ELISAs for both PR3-ANCA and MPO-ANCA, and fluorescence positivity is subjective whereas ELISA results are not. These results suggest the fluorescence detected by commercial slides but not by the smears was either not clinically important or, in the case of the P-ANCA, was not from a patient with Wegener's granulomatosis or microscopic polyangiitis. Washed peripheral blood smears have been used successfully for the past 15 years by the laboratory that first described ANCA, ${ }^{1}$ which performs the most ANCA screening tests in Australia.

The patterns and intensities of all fluorescent sera were similar when washed peripheral smears and commercial neutrophil preparations were compared.

The results of testing 500 sera for ANCA with washed peripheral blood cells showed that the proportion of C-ANCA sera with PR3 specificity was similar to that described elsewhere using in house neutrophil cytospin smears. ${ }^{7}$ Furthermore C-ANCA and P-ANCA values demonstrated by IIF correlated well with PR3- 

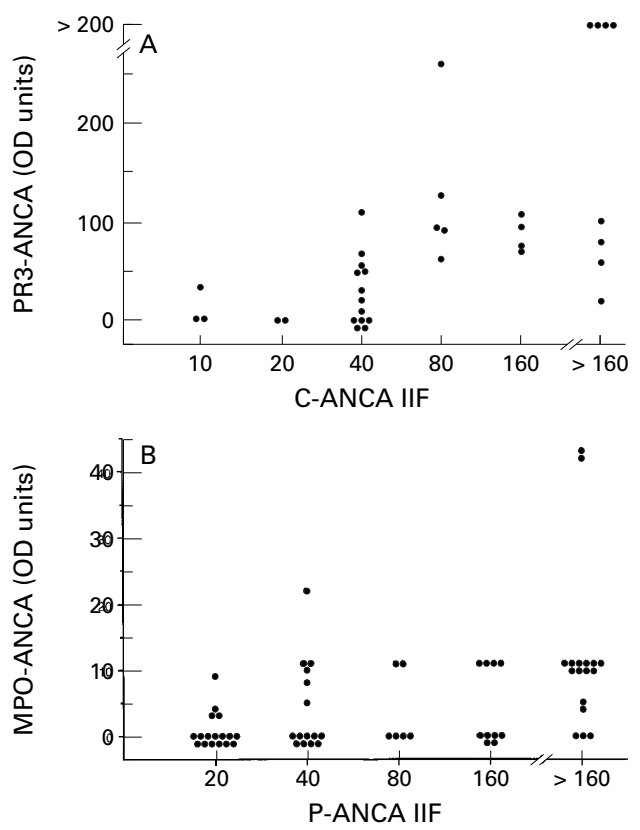

Figure 2 (A) Correlation between PR3-ANCA binding in ELISA and $C-A N C A$ fluorescence intensity; (B) correlation between MPO-ANCA binding in ELISA and $P$-ANCA fluorescence intensity. ANCA, antineutrophil cytoplasmic antibodies; $C-A N C A$, cytoplasmic; $P-A N C A$, perinuclear; IIF, indirect immunofluorescence; $M P O$ myeloperoxidase; $O D$, optical density; PR3, proteinase 3.

ANCA and MPO-ANCA values, respectively, which has not been shown previously. ${ }^{8}$

Few sera that were IIF negative using washed peripheral blood cells were positive in either
PR3-ANCA or MPO-ANCA ELISAs, suggesting a low "false negative" rate for ANCA. However, this method may detect more "false positive" ANA, because sera are screened for ANCA at a 1/10 dilution, which will demonstrate low amounts of nuclear fluorescence that cannot be confirmed at a $1 / 40$ dilution on HEp2 cells. Because some of these ANA might mask an ANCA, this will result in more sera being tested in ELISAs for both PR3-ANCA and MPO-ANCA according to the International consensus statement guidelines.

1 Davies DJ, Moran JE, Niall JF, et al. Segmental necrotising glomerulonephritis with antineutrophil antibody: possible arbovirus aetiology? BMf 1982;285:606-7.

2 van der Woude FJ, Rasmussen N, Lobatto S, et al. Autoantibodies against neutrophils and monocytes: tool for diagnosis and marker of disease activity in Wegener's granulomatosis. Lancet 1985;i:425-9.

3 Savage COS, Winearls CG, Jones S, et al. Prospective study of radioimmunoassay for antibodies against neutrophil cytoplasm in diagnosis of systemic vasculitis. Lancet 1987; i: $1389-93$.

4 Savige J, Gillis D, Davies D, et al. International consensus statement on testing and reporting of antineutrophil cytoplasmic antibodies (ANCA). Am f Clin Pathol 1999; 111:507-13.

5 Wiik A. Delineation of a standard procedure for indirect immunofluorescence of ANCA. APMIS 1988;97:(suppl 6): 12 .

6 Wiik A, Rasmussen N, Wieslander J. Methods to detect autoantibodies to neutrophilic granulocytes. In: Manual of biological markers of disease. The Netherlands: Kluwer Academic Publishers, 1993:1-14,

7 Wong RC, Silvestrini RA, Savige JA, et al. Diagnostic value of classical and atypical cytoplasmic antibody (ANCA) immunofluorescence patterns. F Clin Pathol 1999;52:124-8.

8 Wang G, Csernok E, de Groot K, et al. Comparison of eight commercial kits for quantitation of antineutrophil cytoplasmic antibodies (ANCA). I Immunol Methods 1997;208. 203-11. 